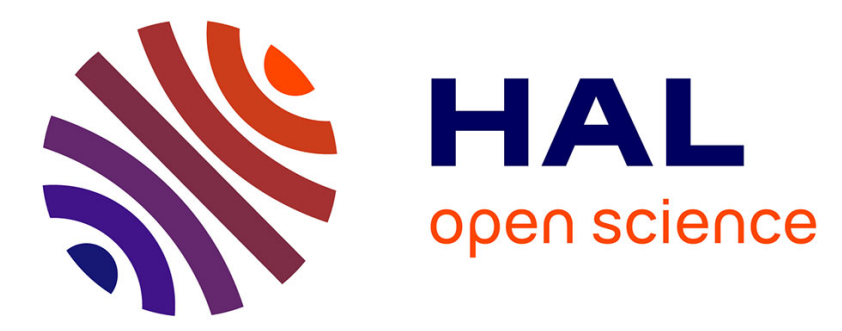

\title{
Convection de Rayleigh-Bénard dans une cavité poreuse faiblement inclinée
}

\author{
J.P. Walch, B. Dulieu
}

\section{To cite this version:}

J.P. Walch, B. Dulieu. Convection de Rayleigh-Bénard dans une cavité poreuse faiblement inclinée. Journal de Physique Lettres, 1982, 43 (4), pp.103-107. 10.1051/jphyslet:01982004304010300 . jpa00232016

\section{HAL Id: jpa-00232016 https://hal.science/jpa-00232016}

Submitted on 1 Jan 1982

HAL is a multi-disciplinary open access archive for the deposit and dissemination of scientific research documents, whether they are published or not. The documents may come from teaching and research institutions in France or abroad, or from public or private research centers.
L'archive ouverte pluridisciplinaire HAL, est destinée au dépôt et à la diffusion de documents scientifiques de niveau recherche, publiés ou non, émanant des établissements d'enseignement et de recherche français ou étrangers, des laboratoires publics ou privés. 


\title{
LE JOURNAL DE PHYSIQUE-LETTRES
}

J. Physique - LETTRES 43 (1982) L-103 - L-107

15 FÉVRIER 1982, PAGE L-103

Classification

Physics Abstracts

47.25Q

\section{Convection de Rayleigh-Bénard dans une cavité poreuse faiblement inclinée}

\author{
J. P. Walch et B. Dulieu \\ Laboratoire de Thermodynamique des Milieux Ioniques et Biologiques, \\ Université Paris VII, Tour 33-43-E2, 2, place Jussieu, 75251 Paris Cedex 05, France
}

(Reçu le 4 novembre 1981, accepté le 21 décembre 1981)

\begin{abstract}
Résumé. - La convection dans une cavité poreuse peu inclinée présente les propriétés d'une transition de phase du premier ordre : divergence de la susceptibilité à l'inclinaison, états multiples et effets d'hystérésis. L'équation de la fronce dans l'espace $\left(R, \varphi, T_{1}\right)$ est donnée et ses conséquences sont comparées aux résultats d'expériences numériques.
\end{abstract}

\begin{abstract}
Convection in a slightly tilted porous cavity is equivalent to a first order phase transition : susceptibility to inclination is divergent, multiple states and hysteresis effects are shown. The equation of the cusp in the space $\left(R, \varphi, T_{1}\right)$ is computed and its consequences are compared with the results of numerical experiments.
\end{abstract}

1. Introduction. - La transition entre régime purement conductif et régime convectif dans les conditions du problème de Rayleigh-Bénard a souvent été comparée à une transition de phase. Nous étudions ici ce qu'il advient d'une telle analogie lorsque la couche fluide est légèrement inclinée sur l'horizontale et, plus particulièrement, lorsque ce fluide sature un milieu poreux homogène.

L'écoulement est alors décrit par la loi de Darcy, soit en régime stationnaire :

$$
\mathbf{V}=\frac{\kappa}{v}\left(-\frac{1}{\rho} \nabla p+\mathbf{g}\right)
$$

où $\mathbf{V}$ est la vitesse de filtration du fluide saturant, $v$ sa viscosité cinématique, $\rho$ sa masse volumique et $p$ sa pression. $\kappa$ est la perméabilité du milieu poreux. Nous utilisons l'équation d'état habituelle ainsi que l'approximation de Boussinesq. 
$\mathrm{Au}$ moyen de la fonction de courant habituelle (nous considérons un écoulement bidimensionnel), l'équation de Darcy s'écrit, sous forme adimensionnelle :

$$
\Delta \psi=R(-\sin \varphi \partial z T+\cos \varphi \partial x T)
$$

où

$$
R=\frac{\alpha g 2 \delta T h \kappa}{v \chi}
$$

est le nombre de Rayleigh de filtration. $\alpha$ est le coefficient de dilatation thermique du fluide, $\delta T$ le demi-écart de température, $h$ l'épaisseur de la cavité, $\chi$ le rapport de la conductivité thermique équivalente du milieu poreux à la chaleur volumique du fluide saturant, $T$ est la température du milieu. L'équation de la chaleur est la même qu'en fluide pur :

$$
\Delta T=\partial x \psi \partial z T-\partial z \psi \partial x T .
$$

Les surfaces horizontales sont isothermes et imperméables, les surfaces latérales adiabatiques et imperméables :

$$
\begin{gathered}
\psi=0 \text { en }\left\{\begin{array}{l}
x= \pm 1 \\
z= \pm 1
\end{array}\right. \text { conditions de glissement } \\
T=\mp 1 \text { en } z= \pm 1 ; \quad \frac{\partial T}{\partial x}=0 \text { en } x= \pm k
\end{gathered}
$$

Ces conditions aux limites, compatibles avec l'utilisation de la loi de Darcy, permettent le développement des inconnues en séries trigonométriques. La résolution de ces équations par la méthode de Galerkin a été décrite précédemment [1,2] et ces résultats seront considérés ici comme ceux d'une expérience numérique. Dans le cadre de cette étude qui se restreint au voisinage du nombre de Rayleigh critique $\left(R_{0}=4 \pi^{2}\right.$ en milieu poreux) 24 termes sont conservés dans le développement des inconnues ( $\square$ et $\square$ sur la figure 1 ).

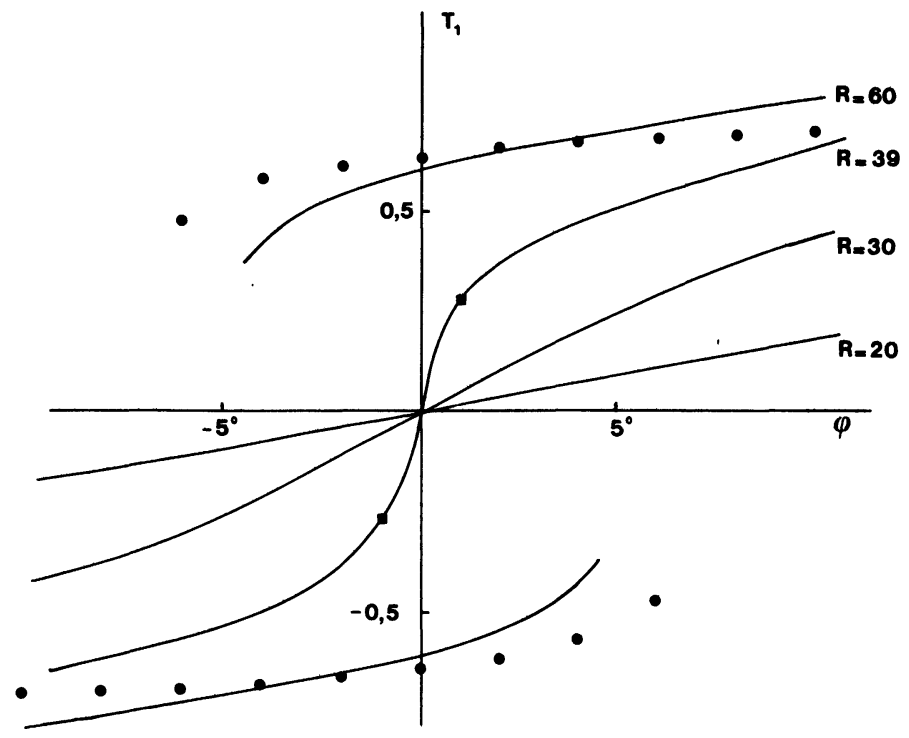

Fig. 1. $-T_{1}$ en fonction de $R:-$ équation (7); $\square$ expériences numériques $(R=39)$; expériences numériques $(R=60)$.

[ $T_{1}$ against $R$.] 
D'autre part, nous partons d'une représentation des champs de température et de vitesse réduite au minimum, ce qui donne, dans le cas de rouleaux carrés :

$$
\begin{aligned}
& T=-z+T_{0} \sin \pi z+T_{1} \sin \frac{\pi x}{2} \cos \frac{\pi z}{2} \\
& \psi=\psi_{1} \cos \frac{\pi x}{2} \cos \frac{\pi z}{2}
\end{aligned}
$$

En négligeant les termes d'ordre supérieur par rapport à l'inclinaison $\varphi$, la résolution approchée des équations de l'énergie et de Darcy donne pour l'amplitude $T_{1}$, au régime stationnaire, l'équation algébrique suivante :

$$
T_{1}^{3}-2^{6} \frac{\left(R-R_{0}\right)}{R^{2}} T_{1}-\frac{2^{11}}{\pi^{3} R} \varphi=0 .
$$

Les solutions de (7) peuvent être considérées comme les extréma d'un potentiel de Landau. Ce sont elles que nous étudions maintenant, en les comparant aux résultats des expériences numériques.

La figure 1 qui donne $T_{1}(\varphi)$ paramétrée par le nombre de Rayleigh, selon (7) et par le calcul numérique, permet de constater que le potentiel(7) constitue une représentation semi-quantitative raisonnable.

2. Susceptibilité à l'inclinaison. - Dans le cas de nombres de Rayleigh nettement sous critiques $(R=20)$, la forme linéarisée de (7) peut être utilisée [3] et l'on obtient une « équation du gaz parfait » :

$$
T_{1}=\frac{8 R}{\pi^{5}} \varphi
$$

Lorsque l'on s'approche des conditions critiques $(R=39)$ l'approximation linéaire n'est justifiée que pour des inclinaisons extrêmement faibles, et fait apparaître une divergence de la susceptibilité à l'origine :

$$
\chi \equiv \frac{\partial T_{1}}{\partial \varphi} \sim \frac{32}{\pi^{3}} \frac{R}{R_{0}-R} \quad \text { pour } \quad R \rightarrow R_{0} .
$$

L'exposant critique $\gamma$ est donc, classiquement, égal à +1 .

3. Etats multiples. - Pour une inclinaison nulle et une valeur de $R$ supérieure à la valeur critique $R_{0}$, on observe deux états convectifs symétriques correspondant aux deux sens de rotation des rouleaux. Au contraire, lorsque la cavité est inclinée, le sens de rotation semble a priori imposé, le fluide montant le long de la paroi chaude et descendant le long de la paroi froide. Appelons " positive " cette circulation « naturelle ".

En fait, comme le montre $\left(^{1}\right)$ l'équation (7), le fluide peut également descendre le long de la paroi chaude : circulation "négative ". Un tel mouvement est thermodynamiquement défavorable, et les deux états correspondant aux mêmes valeurs de $R$ et $\varphi$ sont dissymétriques(Fig. 2) :

$$
\left|T_{1}^{+}\right|>\left|T_{1}^{-}\right| \text {. }
$$

De tels rouleaux « négatifs » sont observables numériquement. Il suffit d'incliner la cellule d'un

( $\left.{ }^{1}\right)$ Pour une inclinaison suffisamment petite. 


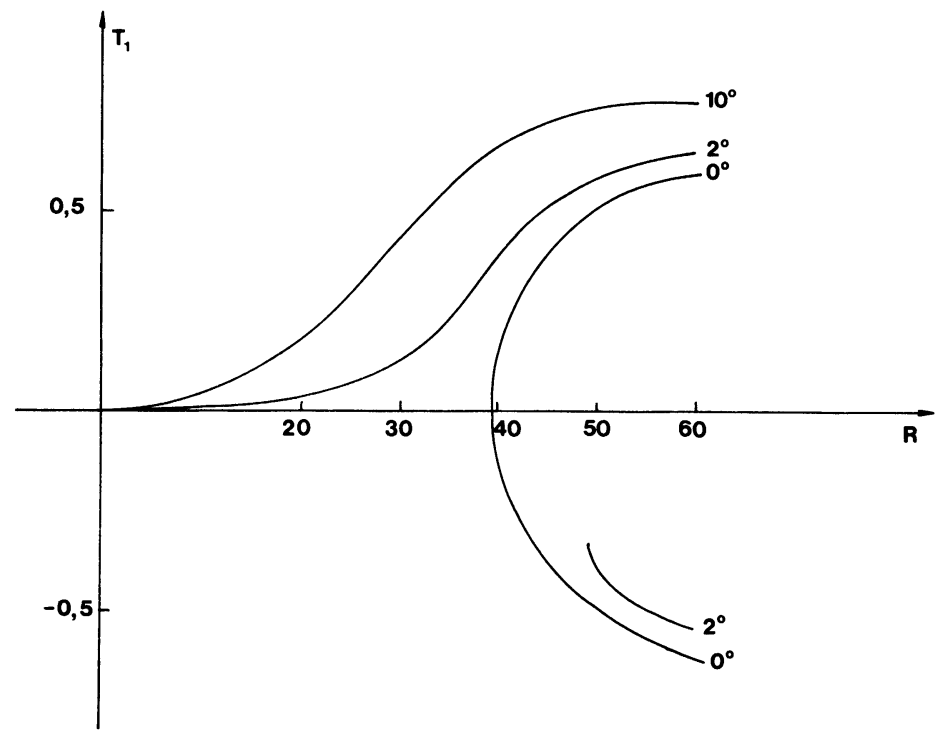

Fig. 2. - Amplitude $T_{1}$ en fonction du nombre de Rayleigh selon (7).

[Amplitude $T_{1}$ against $R$ according to (7).]

angle négatif et d'utiliser la solution obtenue pour initialiser les calculs correspondant à une inclinaison positive. La solution numérique converge alors vers l'état "négatif ".

Les flux de chaleur calculés pour les deux types de rouleaux figurent sur le tableau I. Pour $R=60$, le rouleau contrarotatif est observé jusqu'à une inclinaison dépassant $6^{\circ}$. Selon l'équation (7) l'inclinaison limite serait de $4^{\circ} 4$ et l'on aurait alors :

$$
\left|T_{1}^{-}\right|=\frac{1}{2}\left|T_{1}^{+}\right| \text {. }
$$

Tableau I. - Nombre de Nusselt en fonction de l'inclinaison pour $R=60$.

[Nusselt number against inclination for $R=60$.]

\begin{tabular}{ccc}
$\begin{array}{c}\text { Inclinaison } \\
\text { (degrés) }\end{array}$ & $\begin{array}{c}\text { Nombre de Nusselt } \\
\text { (rouleau positif) }\end{array}$ & $\begin{array}{c}\text { Nusselt } \\
\text { (rouleau négatif) }\end{array}$ \\
\hline $0^{\circ}$ & - & - \\
$1^{\circ}$ & 1,78 & 1,78 \\
$2^{\circ}$ & 1,82 & 1,73 \\
$3^{\circ}$ & 1,86 & 1,68 \\
$4^{\circ}$ & 1,90 & 1,63 \\
$5^{\circ}$ & 1,93 & 1,56 \\
$6^{\circ}$ & 1,96 & 1,48 \\
$7^{\circ}$ & 1,99 & 1,34 \\
& 2,02 & pas de rouleau contrarotatif
\end{tabular}

4. Conclusion. - La prise en considération de l'inclinaison de la couche poreuse permet de développer l'analogie bifurcation thermo-convective-transition de phase. Le schéma classique, basé sur un potentiel de Landau, et donnant naissance à une catastrophe de Riemann-Hugoniot 
(fronce dans l'espace $R, \varphi, T_{1}$, cf. [4]) rend compte de manière satisfaisante des propriétés les plus remarquables de ce système : divergence de la susceptibilité à l'inclinaison et existence de solutions multiples avec effets d'hystérésis. Il s'agit bien d'une transition du premier ordre.

A l'heure actuelle, les seules expériences réalisées sur ce problème sont numériques et le problème se pose de la possibilité d'observation réelle des résultats obtenus. En effet, dans les conditions les plus générales, l'écoulement dans une cavité poreuse inclinée devient tridimensionnel [5]. Dans certaines conditions seulement - discutées par ces auteurs - il reste bidimensionnel. Ce point appelle une étude particulière. Avec des conditions expérimentales bien choisies il doit cependant être possible d'observer les deux états dissymétriques prévus.

\section{Bibliographie}

[1] WalCh, J. P., Thèse de 3e cycle, Paris (1979).

[2] Walch, J. P., Dulieu, B., J. Physique 41 (1980) 1245.

[3] WalCh, J. P., Dulieu, B., Int. J. Heat Mass Transfer 22 (1979) 1607.

[4] Тном, R., Stabilité structurelle et morphogénèse (Interéditions, Paris) 1977, p. 63.

[5] Caltagirone, J. P., Bories, S., C.R. Hebd. Séan. Acad. Sci. 291 B (1980) 197. 